\title{
A data science-based analysis of seasonal patterns in outpatient presentations due to olfactory dysfunction*
}

\author{
Jörn Lötsch ${ }^{1,2}$, Thomas Hummel ${ }^{3}$ \\ 'Institute of Clinical Pharmacology, Goethe - University, Frankfurt am Main, Germany \\ 2 Fraunhofer Institute of Molecular Biology and Applied Ecology - Project Group Translational Medicine and Pharmacology (IME- \\ TMP), Frankfurt am Main, Germany \\ ${ }^{3}$ Smell and Taste Clinic, Department of Otorhinolaryngology, TU Dresden, Dresden, Germany
}

Rhinology 58: 2, 151- 157, 2020

https://doi.org/10.4193/Rhin19.099

*Received for publication:

February 22, 2019

Accepted: September 21, 2019

\begin{abstract}
Background: Changes in human olfactory function throughout the year appear to be a common perception due to the seasonal oscillations in some etiologies associated with olfactory loss. However, longitudinal data from large cohorts were rarely analysed for temporal patterns of human olfaction apart from oscillations in specific etiologies of olfactory loss.

Methods: Temporal patterns in the presentation of patients with olfactory disorders to a single centre were investigated as part of a cohort study. The time series analysis performed utilized a power spectrum analysis and an autoregressive integrated moving average (ARIMA) model in order to demonstrate repetitive fluctuations or trends in the monthly number of patients reporting from January 1999 to December 2017. The analyses additionally addressed temporal changes in the causes to which the olfactory disorder was attributed and in the degree of olfactory loss.
\end{abstract}

Results: A cohort of 7,014 patients was included. Descriptive analysis showed that the presentation of olfactory disorders had seasonal variation, high in March, without a trend. Power spectrum analysis showed a general seasonality of the numbers of patients, without further pattern in the causes or the degree of olfactory dysfunction.

Conclusions: The yearly periodicity in patient presentations at a specialized smell and taste clinic, was not readily attributable to seasonally changing medical causes of olfactory loss such as viral infections. This suggests that in addition to exploring the seasonality of olfactory etiologies, the changes in human olfactory acuity merit further assessments in longitudinal studies..

Key words: data science, time series analytics, computational biomedical research, olfaction, human, ENT clinic

\section{Introduction}

As many biological processes, human olfaction has been shown to display temporal patters such as a circadian rhythm ${ }^{(1,2)}$. Changes in human olfactory function throughout the year also appear to be a common observation; however, they are mostly attributed to the seasonal oscillations in viral infection or allergy-related rhinitis causing olfactory loss. A PubMed search at https://www.ncbi.nlm.nih.gov/pubmed/ for "(((human) AND olfaction) AND seasonal) NOT review[Publication Type]" on February 15, 2019, found 68 hits. When adding "AND allergic" to the search string, 49 hits remained, and when alternatively adding "AND (influenza OR infect*), seven hits remained. The premise of removing "allergic" articles and then "infect*" may have led to the exclusion of some relevant articles. The remaining hits were either reports unrelated to the search intentions, such as public health -related problems causing olfactory annoyances, with the seasonality of the smell of the human microbiome, with seasonal olfactory performances of insects, seasonal temperatures of wine, or with seasonal changes in the composition of flavours. Seasonal rhinitis was mainly investigated for its pathophysiology ${ }^{(3)}$ or therapy ${ }^{(4)}$, without addressing the seasonality as the topic or research. Similarly, infectious settings of olfactory loss were investigated retrospectively with a focus on an association with a decreased rate of influenza vaccination in a small group 
of 84 subjects ${ }^{(5)}$, without a research focus on the seasonality of olfactory function. Similarly, a seasonality observed in olfactory function was attributed to the seasonality of its infectious cause in an independent earlier assessment ${ }^{(6)}$.

Longitudinal olfactory data from large cohorts in which temporal patterns of human olfaction could be addressed are rarely available if at all. The assessments of large olfaction-related cohorts seem therefore restricted to cross-sectional data. In the present study, a large single-centre cohort of 7,014 patients with olfactory disorders observed during a period of 19 years was investigated for temporal patterns.

\section{Materials and methods}

\section{Study design and setting}

This was an observational cohort study performed in outpatients who had presented at the Smell \& Taste Clinic, Dept. of ORL, TU Dresden, Germany. Collection of patient data during routine diagnostics for future scientific evaluation is approved by the Ethics Committee of the Faculty of Medicine of the TU Dresden (number EK251112006). Written informed consent was obtained from all subjects where they agreed to retrospective analysis of clinical findings and transfer of anonymized data to other academic institutions. The consent form is covered by the aforementioned ethic's approval.

\section{Participants}

In this retrospective study, all outpatients were included who by referral from a doctor or spontaneously had presented at the Smell \& Taste Clinic, Dept. of ORL, TU Dresden, Germany, with the symptom "olfactory loss", between January 1999 and December 2017. Inclusion criteria were (i) olfactory loss comprising both, quantitative and qualitative olfactory disorders and (ii) spontaneous report or referral by a medical doctor. Exclusion criteria from the present analysis were (i) congenital olfactory dysfunction, (ii) major cognitive deficits or inability to cooperate during olfactory testing. Furthermore, (iii) subjects were excluded who actively responded to flyers or internet advertising to participate in specific studies on human olfactory function that took place at the same time in the same medical facility.

\section{Objectives}

The main objective of the present analysis was the identification of a possible temporal pattern in patient presentations at the Smell and Taste clinic. As secondary objectives, a temporal pattern in the causes of olfactory loss and in the associated olfactory diagnosis were analysed.

\section{Variables and measurements}

Olfactory function was assessed as described previously ${ }^{(7)}$. Specifically, the "Sniffin'Sticks" (8) test battery (Burghart, Wedel, Germany) was used, which uses felt-tip pens filled with solutions of odours that are placed, with the cap removed, for approximately $3 \mathrm{~s}$ at $1-2 \mathrm{~cm}$ beneath the nostrils. The test battery is composed of three different sets of pens for the assessment of (i) odour thresholds, (ii) the performance in odour discrimination and (iii) the performance in odour identification. The level of olfactory function was derived from the sum of the three scores, i.e., $[0, \ldots, 16]$ for discrimination, $[0, \ldots, 16]$ for identification, and $[1, \ldots, 16]$ for thresholds, as follows: Sum scores of TDI (Threshold, Discrimination, Identification) $\geq 30.5$ in males and TDI $\geq 29.5$ in females indicated normal olfactory function ${ }^{(9)}$.

\section{Statistical methods}

Data were analysed using the R software package (version 3.5.2 for Linux; http://CRAN.R-project.org/ (10)) on an Intel Core i9 ${ }^{\circledR}$ computer running on Ubuntu Linux 18.04.1 64-bit).

\section{Quantitative variables}

The retrospective analysis focused on temporal patterns in monthly patient presentations at the Smell \& Taste Clinic, Department of ORL, TU Dresden, Germany, between January 1999 and December 2017. Time series were analysed in (i) the numbers of patients who reported each month at the clinic, (ii) in the monthly incidence of reduced olfactory function, and (iii) in the monthly mixture of specific etiologies to which the olfactory loss was attributed. Time series were explored using (i) descriptive analysis and (ii) power spectrum analysis. In the case of a positive finding, (iii) time series forecasting was added.

\section{Frequency spectrum analysis}

Non-parametric spectral analysis was used to decompose the time series into sine or cosine functions of different frequencies. This aimed at identifying particularly important or predominant frequencies in the time series. Frequency, decomposition was obtained by means of the Fast Fourier Transformation algorithm (FFT ${ }^{(11)}$ ). Periodogram visualization was used to identify the dominant periods (or frequencies) of the time series. To enhance the identification of dominant frequencies, the obtained power spectrum was smoothed, for better visibility, using a modified Daniell kernel as implemented in the basic $\mathrm{R}$ software package. FFT based analysis was applied on the time series of the monthly numbers of patients reporting for olfactory disorders from January 1999 to December 2017. In addition, the monthly proportion of patients for whom non-normal olfactory function was diagnosed were analysed for temporal patterns. Furthermore, it was explored as to whether particular causes of olfactory disorders display seasonal oscillations in their frequency. Therefore, for each of the nine etiologies to which the olfactory disorders had been attributed (Table 1), the relative occurrence among the monthly patient presentations was calculated as the percentage of the total patient count of the respective month. These percentages were submitted, for each aetiology separa- 
Table 1. Demographics of the enrolled subjects, separately for the subjects' sex and the physiological or pathological (aetiology) condition associated with the subjects' olfactory functional acuity.

\begin{tabular}{|c|c|c|c|c|}
\hline \multirow[t]{2}{*}{ Aetiology } & \multicolumn{2}{|c|}{ Men } & \multicolumn{2}{|c|}{ Women } \\
\hline & n (\% within aetiology) & Age (mean $\pm S D^{\#}$ ) [years] & n (\%) & Age (mean \pm SD) [years] \\
\hline CNS $^{*}$ & 49 (1.6 \%) & $53.1 \pm 15.6$ & 91 (2.9\%) & $55.6 \pm 13.9$ \\
\hline Congenital & $118(3.8 \%)$ & $26.4 \pm 15.4$ & $167(5.4 \%)$ & $27.1 \pm 16.4$ \\
\hline Idiopathic & 841 (27 \%) & $60.4 \pm 14.9$ & $976(31.3 \%)$ & $59.3 \pm 14.5$ \\
\hline Sinunasal & $600(19.3 \%)$ & $54.7 \pm 13.5$ & $566(18.2 \%)$ & $53.9 \pm 13$ \\
\hline Neurodegenerative & $88(2.8 \%)$ & $65 \pm 11.7$ & $63(2 \%)$ & $64.8 \pm 11.3$ \\
\hline Normal & $27(0.9 \%)$ & $40.1 \pm 14.2$ & $21(0.7 \%)$ & $41.2 \pm 13.6$ \\
\hline Postviral & $692(22.2 \%)$ & $57.4 \pm 12.7$ & $1468(47.1 \%)$ & $58.8 \pm 12$ \\
\hline Toxic & $50(1.6 \%)$ & $59.2 \pm 12.4$ & $43(1.4 \%)$ & $61 \pm 14.5$ \\
\hline Traumatic & $649(20.8 \%)$ & $46.9 \pm 15$ & $505(16.2 \%)$ & $48.9 \pm 15.9$ \\
\hline
\end{tabular}

* CNS: indicates mixed causes related to central nervous issues, e.g., stroke, herpes encephalitis, meningeomas of the olfactory bulb. " SD denotes the standard deviation.

tely, to power spectral analysis. Furthermore, as a more global measure of the monthly mixture of causes of olfactory disorders, the Shannon-Wiener diversity index ${ }^{(12)}$ and Fisher's a measure (13) were calculated. Finally, the TDI scores were transformed into olfactory diagnoses according to the standard limits described above and submitted to spectral analysis. These calculations were performed using the R library "vegan" (https://cran.r-project.org/package=vegan ${ }^{(14)}$ ).

\section{ARIMA modelling}

Time series forecasting was employed to assess whether temporal patterns found in the data were consistent and suitable to predict future data. Therefore, AIRMA models ${ }^{(15)}$ were created, which contain an auto-regressive (AR), an integrated (I), and a moving average (MA) component to make use of past values, trends or errors, respectively, for forecasting future values. Specifically, a seasonal model was implemented as ARIMA ( $p, d$, $q)(P, D, Q)[S]$, where $p$ and $q$ denote the order of auto-regression and moving average, respectively, and $d$ means the degree of trend differencing in the integrated component, q means the order of moving average. Furthermore, $\mathrm{P}$ and $\mathrm{Q}$ denote the seasonal lags of auto-regression and moving average, $D$ denotes the degree of seasonal difference, and $\mathrm{S}$ denotes the length of the seasonal component, which was set at a value of $S=12$ considering the focus on the yearly patterns in the monthly patient presentations.

The stationarity of the time series, i.e., time-invariant mean and variance, was checked by means of the Augmented Dickey-Fuller (ADF) unit-root test ${ }^{(16)}$. If necessary, stationarity was achieved by differencing the time series. The parameters of ARIMA model were estimated based on the exploration of autocorrelation (ACF) and partial autocorrelation (PACF) graphs of the original and differenced tie series. These calculations were performed using the R library "forecast" (https://cran.rproject.org/package=forecast $\left.{ }^{(17)}\right)$. Specifically, parameters were identified automatically employing the "auto.arima" function of the R library, without and with setting the seasonality parameter to "TRUE", or with setting a seasonal difference to $D=1$. In addition, ARIMA parameters were adapted based on the ACF and PACF plots. The final model was selected on the basis of the ACF and PACF plots of the residuals, the Akaike information criterion ${ }^{(18)}$ and the Box-Ljung test ${ }^{(19)}$, which examines whether there is no remaining autocorrelation among the residuals of the model fit. In addition, only those models were further regarded for which all parameters passed the Wald test ${ }^{(20)}$ as implemented in the "coeftest" function of the R package "Imtest" (https://cran.rproject.org/package=Imtest ${ }^{(21)}$ ) Model building was performed using roughly $80 \%$ of the data while the forecasting performance was tested at the remaining $20 \%$.

\section{Results}

\section{Participants and descriptive data}

A total of 7014 subjects were included in the analysis with an age range of 5 to 95 years (mean $55 \pm 15.7$ ) and with being 3114 male and 3900 female was included in the analysis. Patients were grouped according to their medical diagnosis made during evaluation of the olfactory disorder that caused them to seek medical help. Grouping was performed according to the recent position paper on diagnostics and treatment of olfactory dysfunction ${ }^{(22)}$. This could be grouped into nine different etiologies (Table 1).

\section{Outcome data}

All subjects having reported between January and Decem- 


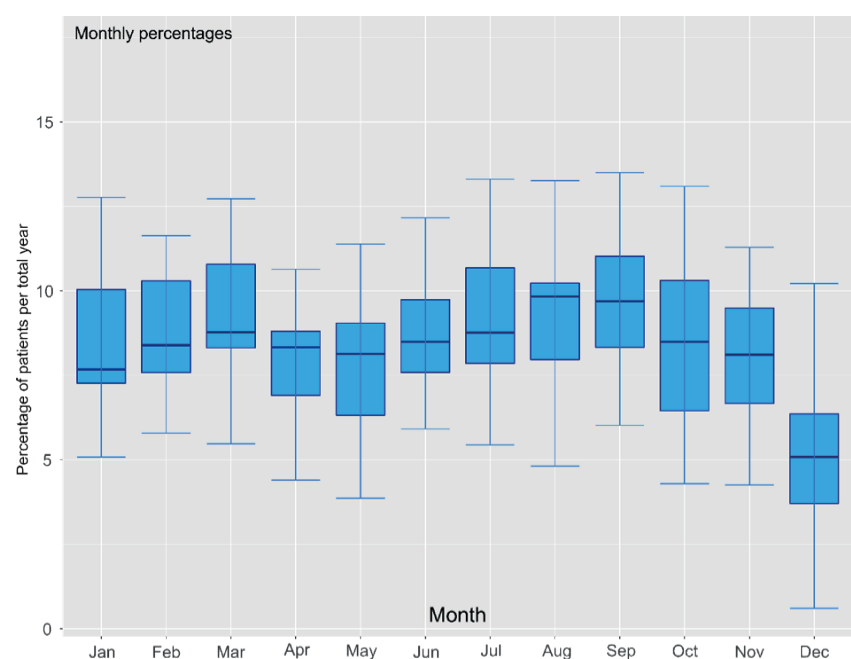

Figure 1. Box and whisker plots showing the monthly number of patients who had reported with olfactory disorders at the Smell \& Taste Clinic between January 1999 and December 2017. The figure has been created using the R software package (version 3.5.2 for Linux; http:// CRAN.R-project.org/ $\left.{ }^{(10)}\right)$ with the libraries "forecast" (https://cran.r-project.org/package=forecast ${ }^{(17)}$ ) and "ggplot2" (https://cran.r-project.org/ package $=$ ggplot 2 .

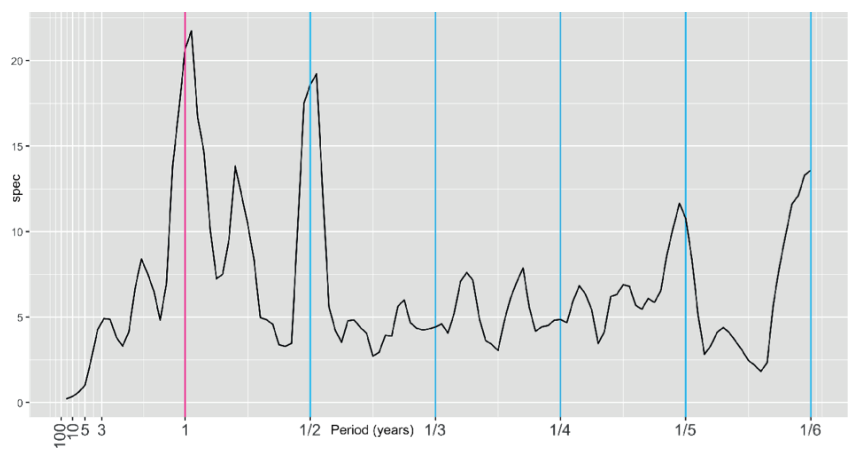

Figure 2. Plot of the results of a Fast Fourier Transformation analysis of the time series regarding the monthly number of patient presentations for olfactory disorders from January 1999 to December 2017. The periodogram plots the spectral power (ordinate) against the periods of the frequencies (abscissa). The maximum power was observed for a frequency with a period of 1 year (red perpendicular line). The figure has been created using the R software package (version 3.5.2 for Linux; http://CRAN.R-project.org/ $\left.{ }^{(10)}\right)$ with the libraries "forecast" (https://cran.rproject.org/package=forecast ${ }^{(17)}$ ) and "ggplot2" (https://cran.r-project. org/package=ggplot 2 . ber 2017 were included in the analysis, which amounted to a cohort size of $n=n=$ subjects. They had reported for olfactory disorders associated to nine different etiologies comprising the central nervous system (CNS) disorders such as stroke, herpes encephalitis, meningeomas of the olfactory bulb $(n=140$ subjects), congenital $(n=285)$, idiopathic $(n=1,817)$, nasal ( $n$ $=1,166)$, neurodegenerative $(n=151)$, normal $(n=48)$, postviral $(n=2,160)$, toxic $(n=93)$ and traumatic $(n=1,154)$. A more detailed report is provided in Table 1. A plot of the monthly numbers of patients who had presented with olfactory disorders at the Smell \& Taste Clinic between January 1999 and December 2017 suggested a seasonal pattern. For example, a clear low was observed in December, and in March number of patients seemed higher than in the neighboured months, whereas an apparent peak in June was just an observation in a single year (Figure 1).

\section{Main results}

\section{Frequency spectra}

Following spectral analysis, the periodogram plot (Figure 2) displayed its maximum at a frequency corresponding a period of 1 year, which indicates the detection of a 12-months rhythm as the main characteristics of the temporal pattern of the monthly numbers of patients who had presented with olfactory disorders at the Smell \& Taste Clinic between January 1999 and December 2017. Additional smaller peaks were observed at the periodogram at localizations that corresponded to oscillations with periods of 0.5 and $1 / 6$ years.
The decrease in patient presentations in December (Figure 1), which was probably due to the local holidays, was interpreted as a possible confounder. To test this whether the observed periodic patterns were just caused by this decrease, the number of reports in December was replaced (i) either with the median monthly number of patient presentations of the remaining 11 months in the respective year, (ii) or with the number of patient report in a randomly chosen other month of the respective year. In both scenarios, the yearly cycle prevailed (details not shown). Only the two additional cycles became less evident than in the original data. Applying the same data replacement to any other month had no such effect, i.e., the periodograms kept their appearance (details not shown). Thus, the low number of patients reporting in December could not be identified as the main cause underlying the yearly cycle of the reports.

The nine different etiologies to which the olfactory deficits were attributed (Table 1) lacked a consistent pattern of their temporal appearance (Figure 3 top four rows). The periodogram plots obtained following spectral analysis lacked typical peaks at the expected periods of $1,1 / 2$ or $1 / 4$ year. Similarly, the diversity of etiologies with which the patients presented each month, numerically described by either the Shannon-Wiener diversity index (Figure 3 bottom left panel) or by Fisher's a measure (not shown) did not display a pattern that would have supported a consistent seasonal variation. Finally, the olfactory diagnosis of non-normal function, which is based on the TDI score as described in the methods section, did not show any frequency pattern that would have pointed at a seasonal cycle (Figure 3 bottom 

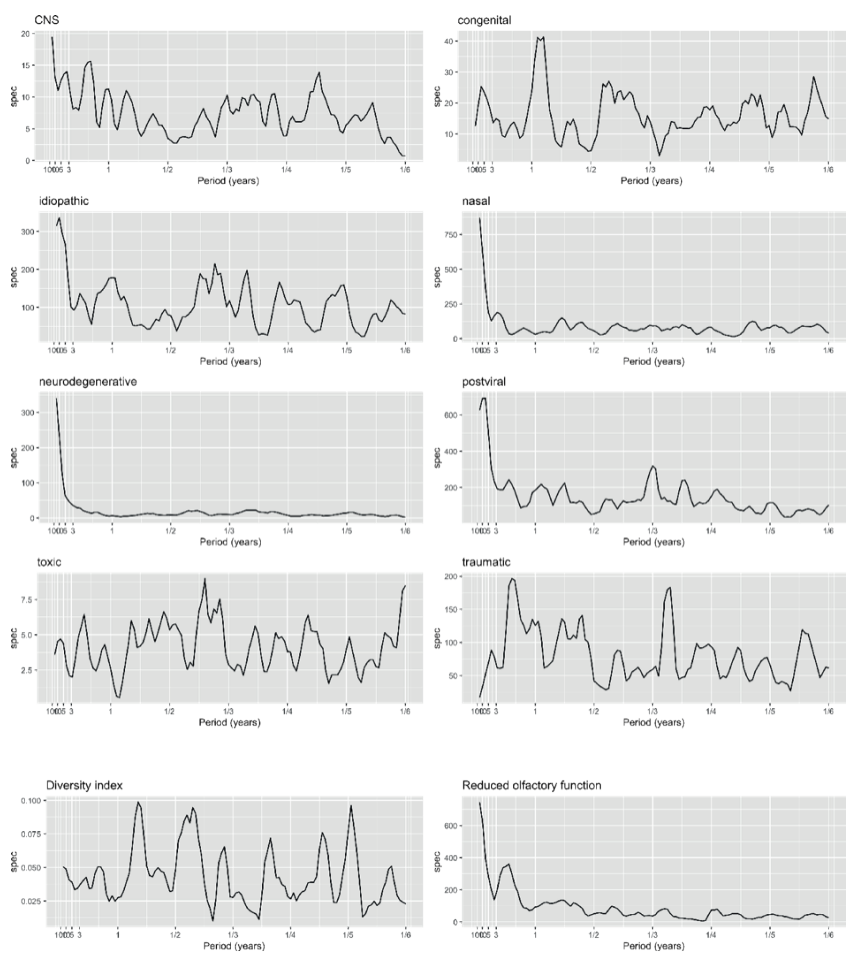

Figure 3. Plot of the results of a Fast Fourier Transformation analysis based periodogram of different time series, i.e., (i) the percentage of each aetiology to which olfactory disorders were attributed (top four rows), (ii) the monthly diversity of cases to which the olfactory loss was attributed (bottom left panel) and (iii) the monthly fraction of reduced olfactory function (bottom right panel). The periodograms plot the spectral power (ordinate) versus the periods of the frequencies (abscissa). The figure has been created using the R software package (version 3.5.2 for Linux; http://CRAN.R-project.org/ $\left.{ }^{(10)}\right)$ with the libraries "forecast" (https://cran.r-project.org/package=forecast ${ }^{(17)}$ ) and "ggplot2" (https:// cran.r-project.org/package=ggplot 2 .
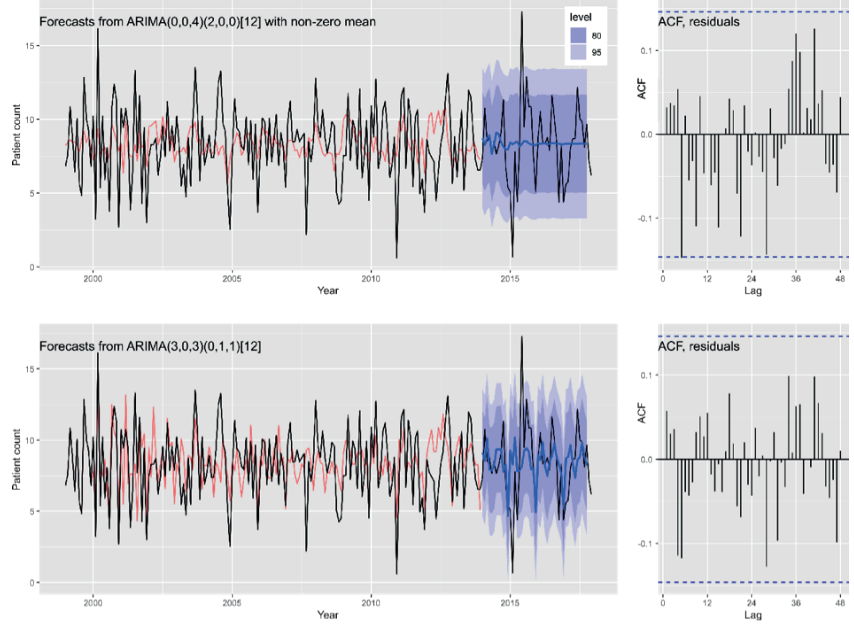

Figure 4. Fit of the time series regarding the monthly number of patient presentations for olfactory disorders, using (i) the automatic model estimation implemented as "auto.arima" in the R library "forecast" (17) (upper line of plots), and (ii) an $\operatorname{ARIMA}(3,0,3)(0,1,1)[12]$ model that had emerged as the best among several alternatives tested (lower line of plots). Left panels: The original values are shown as a black line. The ARIMA model had been created using the observation period from January 1999 to December 2013. The fitted values are shown as a red line. The trained model was then used to forecast the number of patient reporting monthly to the Smell \& Taste Clinic between January 2014 and December 2017. The blue line indicates the mean of the forecast, surrounded by the areas limiting the $95 \%$ and $80 \%$ confidence intervals of the forecast. Right panels: Autocorrelation plot of the residuals of the ARIMA model and $95 \%$ significance boundaries (blue dotted lines). The figure has been created using the R software package (version 3.5.2 for Linux; http://CRAN.R-project.org/ $\left.{ }^{(10)}\right)$ with the libraries "forecast" (https:// cran.r-project.org/package=forecast ${ }^{(17)}$ ) and "ggplot2" (https://cran.rproject.org/package=ggplot 2 . right panel). Thus, FFT based analyses of the frequency patterns in the monthly patient presentations resulted in a general seasonality of the numbers of patients who report, without a further pattern in the causes or the degree of olfactory dysfunction. Monthly report counts were therefore further analysed.

\section{ARIMA models}

Decomposing the time series monthly numbers of patients having reported from January 1999 to December 2014 indicated a clear seasonal pattern without a clear non-stationary trend. Hence, the ADF test was statistically significant $(p=0.01)$. Estimation of the ARIMA using the automatic algorithm provided in the R library "forecast", without or with setting the "seasonal" parameter to "TRUE" resulted in a ARIMA $(0,0,4)(2,0,0)[12]$ model, whereas running the "auto.arima" command with a fixed $D=1$ resulted in an $\operatorname{ARIMA}(0,0,0)(0,1,1)[12]$ model, i.e., a model that contained only seasonal effects. For setting ARIMA parameters, ACF and PACF plots provided no clear-cut identification of the most suitable set; however, a decline after three cycles in the local plots and a peak at 12 cycles in the differenced plots suggested setting $p$ and $q$ to values of 3 and setting $P$ and $Q$ to values of 1 .

A total of seven models was tested to make the results more dependable. All models met the requirements of white noise of residual time series (Box-Ljung tests: all $p>0.05$ ). However, the automatically recognized model $\operatorname{ARIMA}(0,0,4)(2,0,0)[12]$ did not meet the selection criterion because of the highest AIC value and provided a poor forecast (Figure 4 upper line). ARIMA $(3,0,3)$ $(0,1,1)[12]$ (Figure 4 lower line) had the lowest AIC and was selected as the best ARIMA model of this analysis. All of its parameters were differed statistically significantly from zero as indicated by the results of the Wald test, which for the values of $\mathrm{p} 1=$ $-1.131, \mathrm{p} 2=0.6713, \mathrm{p} 3=0.8269, \mathrm{q} 1=0.9830, \mathrm{q} 2=-0.9817, \mathrm{q} 3=$ -0.9994 , and $\mathrm{Q} 1=-0.8592$ obtained $\mathrm{p}$-values ranging between $3.19 \cdot 10^{-14}$ and $<2.2 \cdot 10^{-16}$, and with confidence intervals of parameter estimates that did not include the value of zero. While 
the ARIMA model was built using data from January 1999 to December 2013, a forecast was possible for the monthly numbers of patients having reported from January 2014 to December 2017 (Figure 4). Hence, the results of the ARIMA supported the findings obtained with FFT and hinted at true seasonal pattern when patients consulted a specialist due to olfactory loss.

\section{Discussion}

Key results

The presentations of patients at a specialized smell and taste center due to olfactory loss showed a temporal pattern with a one-year period. This was not due to the holiday-associated decease in December patient presentations. Compensating for the December-low changed the power spectrum of the time series, however, not its one-year periodical component. The results of a one-year temporal pattern in patient presentations are supported by the ARIMA $(3,0,3)(0,1,2)[12]$ model, which included in its $\mathrm{D}=1$ component the yearly rhythm. All tested ARIMA models with parameter $D=0$ showed substantially higher values of the AIC. The implementation of a forecasting model mainly aimed at providing further support for a temporal pattern, rather than to provide a general model of patient presentations at an olfactory clinical centre.

A possible interpretation of the clearly observed one-year rhythmic pattern in patient presentations during the 19-year observation period may involve that olfactory complaints following a viral infection of the upper respiratory tract had occurred at a seasonal rhythm. However, the relative proportion of post-viral causes among all different causes attributed to the olfactory loss did not change with a temporal pattern. This was also true for the other eight different etiologies attributed to the olfactory loss although for some of them a temporal pattern was not expected anyway such as for congenital anosmia. This contrasts with an earlier report of a peak incidence of postviral anosmia in March found in an analysis of a six-year period (6). In that analysis, the absolute incidence had been analysed in contrast to the present analysis, where the incidence of each aetiology of olfactory loss was analysed at a relative level. Hence, the present analysis apparently reproduced that earlier finding as also showing a peak of patient presentations in March; however, this peak could not be attributed to postviral olfactory disorders while the accumulation of patients in March seemed to be due to several causes not shifting the mixture of etiologies toward a specific one.

\section{Limitations}

Thus, the findings when compensating for December produced the expected results that this apparent seasonal variation owes to the low number of subjects reporting in December as the clinic is closed during the winter holidays for almost two weeks This does not occur at any other time during the year, except for one or two-day long periods when the unit is closed throughout the year. Indeed, a holiday-based explanation of the higher number in March does not come readily to us, and therefore, we have focused on December. Thus, the lack of clear causes of the yearly pattern in patient presentations may relate to some limitations in this study. Firstly, this was a single-center study performed in a facility specialized in research and treatment of olfactory and gustatory disorders. Patients presented themselves spontaneously or were advised to do so by their physicians. Hence, many patients might have consulted their primary physician first or solely, and therefore did not present at the specialist Smell \& taste centre at the onset of the olfactory loss. Therefore, the monthly incidence of patient presentations analysed in this study might not be identical to the true monthly incidence of olfactory loss in the present geographic region. Moreover, only the variation in patient presentations, in olfactory function and in the attributed causes was considered. A role of other possible factors such as environmental conditions including the weather, or the incidence of influenza were ignored. Therefore, to better understand the seasonal cycles in human olfactory function, future data should be continually acquired longitudinally. Finally, it needs to be emphasized that the timing of onset not being available in the database is a major limitation. Possibly, the delay is random with respect to the different etiologies of olfactory loss. However, this is uncertain as a presentation with olfactory loss following a viral infection may be prompter than a presentation due with olfactory loss due to a stroke or trauma where the treatment of the basic disease had priority. Therefore, it is conceivable that patterns in olfactory loss would have become clearer when the onset information was available.

\section{Interpretation}

Considering the sparse information about seasonal patterns of human olfactory function, the present analysis of time series in a total of 7,014 reports between January 1999 and December 2017 indicated a yearly periodicity that could be addressed with temporal forecasting using an auto-regressive, integrated moving average model. The present results reflect the observations in the highly specialized environment of a smell and taste clinic. They indicate that seasonal peaks in patient presentations need not be expected with common causes of olfactory loss such as viral infections of the upper respiratory tract. The observed seasonality in patient presentations was not readily attributable to seasonally changing medical causes of olfactory loss such as viral infections, the results suggest that fluctuations in the presentation of olfactory disorders itself merit further assessments in longitudinal studies, rather than exploring just the seasonality of olfactory etiologies as it has been the focus of most previous work on fluctuations in the presentation of olfactory disorders. If a particular time series behaviour of human olfactory function could be detected, this might impact on the revision of normal 
values that may possibly need adaptation for seasonal effects.

\section{Generalisability}

The present results provide a basis for planning seasonal peaks in patient presentation at a specialized olfactory health care centre. The limitations of the study such as lack of recording of environment factors, e.g., the weather conditions, or the absence of specific information about the onset of the olfactory loss may provide guidance for the planning of future studies on the seasonal patterns in olfactory disorders.

\section{Acknowledgements}

This work has been funded by the Landesoffensive zur Entwicklung wissenschaftlich - ökonomischer Exzellenz (LOEWE),
LOEWE-Zentrum für Translationale Medizin und Pharmakologie (JL). We also would like to thank the Deutsche Forschungsgemeinschaft for support (DFG HU 441/18-1 to TH and DFG Lo $612 / 10-1$ to JL). The funders had no role in study design, data collection and analysis, decision to publish, or preparation of the manuscript.

\section{Authorship contribution}

$\mathrm{JL}$ : designed and pefomed the anaylses, wrote and revised the manuscript. TH: provided data, helped to design study, revision of manuscript.

\section{Conflict of interest}

The authors have declared that no competing interests exist.

\section{References}

1. Nordin S, Lotsch J, Murphy C, et al. Circadian rhythm and desensitization in chemosensory event-related potentials in response to odorous and painful stimuli. Psychophysiology 2003; 40:612-619.

2. Lötsch J, Nordin S, Hummel T, et al. Chronobiology of nasal chemosensitivity: Do odor or trigeminal pain thresholds follow a circadian rhythm? ChemSenses 1997; 22:593-598.

3. Becker S, Pflugbeil C, Groger M, et al Olfactory dysfunction in seasonal and perennial allergic rhinitis. Acta Otolaryngol 2012; 132:763-768.

4. Higaki T, Okano M, Makihara S, et al. Early interventional treatment with intranasal corticosteroids compared with postonset treatment in pollinosis. Ann Allergy Asthma Immunol 2012; 109:458-464.

5. Flanagan CE, Wise SK, DelGaudio JM, et al. Association of decreased rate of influenza vaccination with increased subjective olfactory dysfunction. JAMA Otolaryngol Head Neck Surg 2015; 141:225-228.

6. Konstantinidis I, Haehner A, Frasnelli J, et al. Post-infectious olfactory dysfunction exhibits a seasonal pattern. Rhinology 2006; 44:135-139.

7. Lötsch J, and Hummel T. A machine-learned analysis suggests non-redundant diagnostic information in olfactory subtests. IBRO Rep 2019; 6:64-73.

8. Hummel T, Sekinger B, Wolf SR, et al. 'Sniffin' sticks': olfactory performance assessed by the combined testing of odor identification, odor discrimination and olfactory threshold. Chem Senses 1997; 22:39-52.

9. Hummel T, Kobal G, Gudziol H, et al. Normative data for the "Sniffin' Sticks" including tests of odor identification, odor discrimination, and olfactory thresholds: an upgrade based on a group of more than 3,000 subjects. Eur Arch Otorhinolaryngol 2007; 264:237-243.

10. R Development Core Team. R: A Language and Environment for Statistical Computing. 2008.

11. Cooley JW, and Tukey JW. An Algorithm for the Machine Calculation of Complex Fourier Series. MathComput 1965; 19:297-301.

12. Shannon CE. A mathematical theory of communication. Bell Syst Techn J 1951; 30:50-64.

13. Fisher RA, Corbet AS, and Williams CB. The relation between the number of species and the number of individuals in a random sample of an animal population. J Animal Ecol. 1943:42-58.

14. Oksanen J, Blanchet FG, Friendly $M$, et al. vegan: Community Ecology Package, 2018.

15. Box GEP, and Jenkins GM. Time series analysis: forecasting and control. Rev. ed., 1976.

16. Said SE, and Dickey DA. Testing for unit roots in autoregressive-moving average models of unknown order. Biometrika 1984; 71:599-607.

17. Hyndman RJ, and Khandakar Y. Automatic Time Series Forecasting: The forecast Package for R. J. Stat Soft. 2008; 1, 3,
18. Akaike H. A new look at the statistical model identification. IEEE Trans Aut Control 1974; 19:716-723.

19. Ljung GM, and Box GEP. On a measure of lack of fit in time series models. Biometrika 1978; 65:297-303.

20. Wald A. Tests of statistical hypotheses concerning several parameters when the number of observations is large. Transactions of the American Mathematical society 1943; 54:426-482.

21. Zeileis A, and Hothorn T. Diagnostic Checking in Regression Relationships. $\mathrm{R}$ News 2002; 2:7-10.

22. Hummel T, Whitcroft KL, Andrews $P$, et al. Position paper on olfactory dysfunction. Rhinol Suppl 2017; 54:1-30.

Prof. Dr. Dr. Jörn Lötsch

Goethe - University

Theodor - Stern - Kai 7

60590 Frankfurt am Main

Germany

Phone: +49-69-6301-4589

Fax: +49-69-6301-4354

E-mail: j.loetsch@em.uni-frankfurt.de 